\title{
Smoking Cessation and Pharmacists: An Australian Pharmacy Student Perspective
}

\author{
Luke J. Parsons \\ Bachelor of Pharmacy (Year IV), University of Sydney, Australia
}

I am a pharmacy student very close to graduating and about to enter practice in a community pharmacy. I would like to describe the pharmacy student experiences before entering the 'real world', and how this training equips us soon-to-be-graduates for dealing with the very real problem of smoking and smoking cessation.

Australia, like many countries, is exposed to an almost constant advertising campaign aimed at encouraging smoking cessation, or more specifically, outlining the dangers of smoking. As a result of this, Australian smokers are now faced with graphic images emblazoned on all cigarette packets and television advertisements warning of the drastic, but probable outcomes of a lifetime of smoking. It is known that in this country $21 \%$ of the population are chronic smokers, and that these are the more 'hard core' smokers, more resistant to treatment for smoking cessation (ABS, 2005).

Smoking killed 14, 790 Australians in 2004-2005, making it the leading cause of premature death in this country (Collins \& Lapsley, 2008). These figures are much higher than any other preventable cause of death, such as alcohol misuse, drug use, suicide, homicide, motor vehicle accidents and airplane crashes. So it stands to reason why the government has spent millions of dollars in recent years in an effort to promote smoking cessation, and stresses the requirement for pharmacists, as first line contact, to be ready to counsel on appropriate techniques.

It is therefore quite simple to understand how important it is, when smokers come to the pharmacy deciding that they wish to quit, that they are met with a health care professional who is equipped with the most up-to-date, evidence-based approach. As it stands currently, pharmacy undergraduates in this country (and I believe in many others) are only given a very small portion of learning time to smoking cessation. In this author's experience, the only theory given over the course of 4 years was a 2-hour lecture in the final year of study. This is grossly inadequate, considering the level of misinformation that abounds in community pharmacy today. Indeed, upon questioning pharmacy staff anecdo- tally, they reported to me that the main source of their education on Nicotine Replacement Therapy (NRT) for example was from the drug company responsible for their manufacture. Whilst learning timetables at universities are basically at a maximum, extra-curricular and even postgraduate opportunities would be invaluable places to provide extra training. It seems to this author that NRT and other smoking cessation pharmacotherapies is 'glossed over' in the curriculum as it seems to warrant less importance when compared to higher scheduled medicines.

Quitting is now very much 'pharmacy turf' and it is the role of the Pharmacist to provide accurate and consistent information to customers to ensure that all relevant information gets to the patient and that pharmacies can maintain the clinical edge to practice. Ironically, the down-scheduling of NRT to increase its availability seems to have resulted in pharmacy staff treating it with less significance than from when it was prescription only. One way to fix this would be to provide 'clinical updates' to the pharmacists and pharmacy staff in the community in the form of continuing professional education, as we do for almost every other topic in healthcare through organisations such as the PSA.

If nothing else, the lack of consistency of advice presents a very disjointed professional face. It is common practice for customers in this day to visit multiple pharmacies, I myself having heard many customers say 'But I was told this by 'so-and-so'. To illustrate, many patches say on the packaging 'Any smoking while using [the patch] will totally defeat your purpose' or words to similar effect (e.g., the Nicorette ${ }^{\circledR}$ Comsumer Medicine Information). This has often been interpreted as 'Do not smoke while using patches'. Whilst many practicing pharmacists are aware that this is not true, it is shocking to a lot of today's pharmacy students (through this author's experience). Pharmacists and pharmacy students who have not been educated in smoking cessation therapy will read this, and understandably counsel patients accordingly. This makes the job of the smoking 
cessation specialist much more difficult, as before any treatment can be commenced, they first need to break down these barriers created by incorrect information. Conflicting information is a large part of the breakdown in communication on the pharmacists' behalf. While there is no strict pro-forma to smoking cessation, with every patient obviously very different, this breakdown is mainly due to a lack of knowledge of new developments in research. We all work in a rapidly changing system and not remaining vigilant can have potentially disastrous effects.

Very little research or work has been done in this area, looking specifically at smoking cessation in community pharmacy. While it has been brought to my attention that there are at least two specifically tailored programs, it would be true to say that I personally have never seen an advertisement of any kind at any point in my 6 years involved with the profession, including working in a pharmacy chain that offers one of these programs. It is this author's hope that these programs become as well advertised and known as other pharmacy-based programs such as the myriad of weight loss programs, given the already stated impact of smoking on our society.

More research must be done to define the role of the community pharmacist and their assistants (who are more often than not the first point of call for patients) and how they can receive adequate training to achieve the role of a competent interventionist. Pharmacists are finding themselves used more and more as health care professionals and not just 'glorified dispensary technicians'. This is by no means a new development, but it is our ability (and our expectation) to take on these extra responsibilities that allow us to develop our role, and the diligence with which we do this that proves our worth as a profession.

\section{References}

Australian Bureau of Statistics. (2004/2005). Tobacco smoking in Australia: A snapshot, 2004-05. Available at http://www.abs.gov.au/AUSSTATS/abs@.nsf/mf/4831.0.55. 001? OpenDocument

Collins, D., \& Lapsley, H. (2008). The costs of tobacco, alcohol and illicit drug abuse to Australian society in 2004-05. Canberra, Australia: Department of Health and Ageing. 